\title{
Performance of Coarse and Fine Timing Synchronization in OFDM Receivers
}

\author{
Ali A. Nasir \\ School of Engineering, CECS, \\ The Australian National University \\ Canberra, Australia. \\ ali.nasir@anu.edu.au
}

\author{
Salman Durrani \\ School of Engineering, CECS, \\ The Australian National University \\ Canberra, Australia. \\ salman.durrani@anu.edu.au
}

\author{
Rodney A. Kennedy \\ School of Engineering, CECS, \\ The Australian National University \\ Canberra, Australia. \\ rodney.kennedy@anu.edu.au
}

\begin{abstract}
The performance of OFDM receivers is very sensitive to timing synchronization errors. This paper makes an analysis of different proposed timing synchronization algorithms, their training symbol patterns and their effect on the performance of OFDM systems under severe frequency selective Rayleigh fading. We show BER and MSE performance of six popular preamble based algorithms using joint synchronization and channel estimation to make an insightful and thorough comparison. We analyze the performance with both coarse and fine timing recovery and show that BER performance with fine timing is better for every algorithm. We propose a new technique for timing synchronization that uses Constant Amplitude Zero Auto Correlation (CAZAC) sequences to acquire unit Peak to Average Power Ratio (PAPR) for the preambles. We show that the proposed technique is robust under high delay spread environments with BER and MSE performance comparable to the best case.
\end{abstract}

\section{INTRODUCTION}

Orthogonal Frequency Division Multiplexing (OFDM) has gained enormous popularity for both wire line and wireless communication and broadcasting systems such as wireless local area networks, wireless metropolitan area networks and digital video broadcasting systems [1]. By transmitting multiple modulated sub-carriers in parallel, with each sub-carrier occupying a narrow bandwidth, OFDM can achieve high data rate transmission and robustness to multipath delay spread. However, the performance of OFDM receivers is very sensitive to timing synchronization. Loss of synchronization introduces Inter Carrier (IC) and Inter Symbol Interference (ISI) and can severely degrade the performance of OFDM receivers [2], [3].

Many synchronization techniques have been proposed in literature for OFDM receivers [4]-[13]. Most techniques are training based and exploit the periodic nature of the time domain pilot symbols [4]-[8]. Other techniques exploit the correlation between the cyclic prefix and its data replica [9], [10], but their performance is degraded in multipath environments. In [11], a technique independent of preamble pattern is proposed, which can be applied to OFDM systems with any preamble structure. Blind techniques have also been proposed which do not rely on cyclic prefix or training information [12], [13]. However the processing delay and computational complexity of these algorithms is high, making them unfeasible for high delay spread environments. For both correlation and training based approaches, some techniques allow for a coarse timing synchronization to determine the Fast Fourier Transform (FFT) window for each symbol, followed by a fine tuning based on the impact on channel estimation [5], [9], [10]. The performance of an OFDM receiver can be improved by iterating between the synchronization and channel estimation using information in one for the other [14]. A major limitation of previous work, with the exception of [5], is that they do not consider synchronization and channel estimation jointly. This is crucial because errors in synchronization can affect channel estimation and vice versa [2]. In addition, previous work has compared timing synchronization techniques based on Mean Square Error (MSE) performance only. For a more realistic performance comparsion, it is important to consider Bit Error Rate (BER) performance of an OFDM receiver with joint timing synchronization and channel estimation.

In this paper, we propose a new synchronization technique for timing synchronization in OFDM receivers and compare its performance with six popular preamble based timing synchronization algorithms by Schmidl and Cox [4], Minn et al. [5], Park and Cheon [6] , Ren et al. [7], Kang et al. [11] and Yi et al. [8]. The major contributions of this paper, in comparison to previous research, are as follows:-

- We show BER and MSE performance with every algorithm using joint synchronization and channel estimation to make an insightful and thorough comparison.

- We analyze the performance with both coarse and fine timing recovery and show that BER performance with fine timing is better for every algorithm than with only coarse timing.

- We propose a new technique for timing synchronization that uses Constant Amplitude Zero Auto Correlation (CAZAC) sequences to acquire unit Peak to Average Power Ratio (PAPR) for the preambles. We show that the proposed technique is robust under high delay spread environments with BER performance comparable to the best case.

The rest of the paper is organized as follows. Section II describes the system model including the effects of synchronization errors on OFDM systems. Section III summarizes the synchronization schemes of the aforementioned authors. Section IV details the proposed synchronization technique. Performance evaluation and simulation results are provided 
in Section V. Finally conclusions are drawn in Section VI.

\section{System Model}

The samples of the transmitted baseband OFDM signal, assuming ideal Nyquist pulse shaping, can be expressed as [14]

$$
s(k)=\frac{1}{\sqrt{N}} \sum_{n=0}^{N-1} c_{n} e^{j \frac{2 \pi k n}{N}}, \quad-N_{g} \leq k \leq N-1
$$

where $c_{n}$ is the modulated data or subcarrier symbol, $N$ is the number of inverse fast Fourier transform (IFFT) points and $N_{g}$ is the number of guard samples.

We consider a frequency selective multipath channel with path gains $\left\{h_{\ell}: \ell=0,1, \ldots, K-1\right\}$ and the path delays $\left\{\tau_{\ell}\right\}$ respectively. Assuming no carrier frequency offset, the received samples become

$$
r(k)=\sum_{l=0}^{K-1} h_{\ell} s\left(k-\tau_{\ell}\right)+n(k)
$$

where $n(k)$ is the sample of zero-mean complex additive white Gaussian noise process with variance $\sigma_{n}^{2}$.

\section{A. Receiver}

The block diagram of OFDM receiver considered in this paper is shown in Fig. 1. The synchronization parameters to be estimated are the starting time of the FFT window (timing synchronization) and the channel estimate for equalization. The Coarse Timing Point (CTP) is obtained by taking the time running correlation of the segments that are equal in length to single unique portion of training symbol. The correlation will be strongest at the exact timing point under small delay spreads. However, in severe multipath channels, the timing point is shifted due to channel dispersion, so CTP is preadvanced by some samples $\lambda_{c}$. The channel is estimated on pre-advanced timing point during first iteration and then delay of the first actual channel tap is found from the channel estimate. The CTP is fine tuned by evaluating the Fine Timing Point (FTP) as [5]

$$
\mathrm{FTP}=\tau_{o}+\left(\mathrm{CTP}-\lambda_{c}\right)+\lambda_{f}
$$

where $\lambda_{f}$ is the designed preadvancement to reduce the possible ISI and $\tau_{o}$ is the delay of the first actual channel tap.

After timing synchronization, the ML channel estimate $\hat{h_{\ell}}$ is computed on the basis of FTP. Afterwards, the cyclic prefix is removed from each OFDM symbol and its FFT is taken to do receiver pulse shaping. Since the bandwidth of a sub carrier is designed to be smaller than the coherence bandwidth, each sub channel is seen as a flat fading channel which simplifies the channel equalization process. Hence simple zero forcing frequency domain equalization is done on the basis of channel frequency response $\left\{H_{m}\right\}=F F T_{N}\left(\hat{h}_{\ell}\right)$ to mitigate the single amplitude and phase change from the $m^{\text {th }}$ subcarrier [5]. Finally slicing and decoding is done on equalized symbols.

The data is decoded and recovered correctly if the estimated timing point is within the inter symbol interference (ISI) free

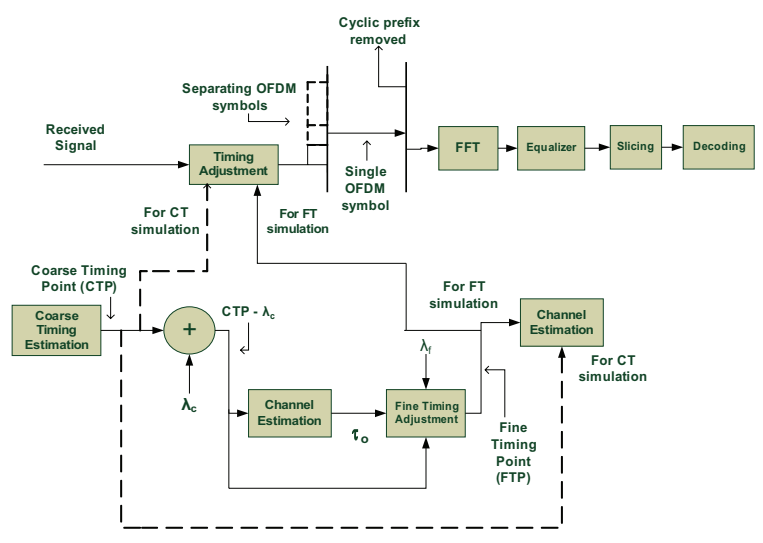

Fig. 1. OFDM Receiver Block Diagram with coarse and fine Timing recovery.

part of the cyclic prefix. Let the sample indexes of a perfectly synchronized OFDM symbol be $\left\{-N_{g}, \ldots,-1,0,1, \ldots, N-\right.$ $1\}$, the timing offset be $\epsilon$, and maximum channel delay spread be $\tau_{\max }$. Then if $\epsilon \in\left\{-N_{g}+\tau_{\max },-N_{g}+\tau_{\max }+1, \ldots, 0\right\}$ (ISI free part), the orthogonality among the sub carriers will not be destroyed and the timing offset will only introduce a phase rotation in every subcarrier symbol $Y_{m}$ at the FFT output

$$
Y_{m}=e^{j \frac{2 \pi m \epsilon}{N}} c_{m} H_{m}+n_{m}, \quad-N_{g}+\tau_{\max } \leq \epsilon<0
$$

where $m$ is the subcarrier index and $n_{m}$ is a complex Gaussian noise term. For a coherent system, this phase rotation is compensated by the channel equalization scheme, which views it as a channel-induced phase shift. If the timing estimate is outside the above range, the orthogonality among the subcarriers will be destroyed due to ISI and intersubchannel interference (ICI) [2]. Channel estimation requires that the channel impulse response seen by the receiver be within the cyclic prefix window on the receiver time scale. If due to the timing offset, some portions of the effective channel are shifted outside this window, these portions cannot contribute to the estimate and channel estimation will suffer an additional error due to this timing offset [2].

\section{SynChronizATION TECHNIQUES}

In this section, we summarize the timing metric, i.e., the normalized correlation sequence, for the six popular training based techniques that have been proposed in literature. In general, the timing metric is defined as

$$
M(d)=\frac{|P(d)|^{2}}{(R(d))^{2}}
$$

where $P(d)$ is the correlation sequence and $R(d)$ is the energy of the received symbol at $d^{\text {th }}$ sample. The strongest point of correlation, i.e., the index of $\max \{M(d)\}$ is forwarded as the coarse timing point.

\section{A. Schmidl \& Cox $(S \& C)$}

In this technique, the training symbol proposed is composed of two identical halves [4]. If $L$ is the number of complex 
samples in one-half of training symbol, then $P(d)$ and $R(d)$ are calculated as

$$
\begin{gathered}
P(d)=\sum_{m=0}^{L-1} r^{*}(d+m) r(d+m+L) \\
R(d)=\sum_{m=0}^{L-1}|r(d+m+L)|^{2}
\end{gathered}
$$

where $r$ is the received signal given by (2) and timing metric $M(d)$ is calcuated using (5).

\section{B. Minn et al.}

In this technique, four identical portions of the training symbol are made by repeating the FFT of quarter length Golay complementary sequence in all the portions. $P(d), R(d)$ and $M(d)$ are given as [5]

$$
\begin{gathered}
P(d)=\sum_{k=0}^{L-2} b(k) \sum_{m=0}^{M-1} r^{*}(d+k M+m) r(d+(k+1) M+m) \\
R(d)=\sum_{i=0}^{M-1} \sum_{k=0}^{L-1}|r(d+i+k M)|^{2} \\
M(d)=\left(\frac{L}{L-1} \frac{|P(d)|}{R(d)}\right)^{2}
\end{gathered}
$$

where $b(k)=p(k) p(k+1), k=0,1, \ldots, L-2 . L$ is the number of identical portions, i.e., 4 and $M$ is the samples in that portion, i.e., 8 .

\section{Park and Cheon}

In this technique, a Pseudo Noise (PN) sequence is used in the training symbol. $P(d)$ and $R(d)$ are given as [6]

$$
\begin{gathered}
P(d)=\sum_{k=1}^{N / 2} r(d+N / 2-k) r(d+k+N / 2-1) \\
R(d)=\sum_{k=1}^{N / 2}|r(d+k-1)|^{2}
\end{gathered}
$$

where $N$ is the FFT length and $M(d)$ is given by (5).

\section{Ren et al.}

In this technique, the constant envelope preamble from the FFT of a CAZAC sequence issued for the training symbol [15]. $P(d)$ and $R(d)$ are given as [7]

$$
\begin{gathered}
P(d)=\sum_{k=0}^{N / 2-1} s_{k} s_{k+N / 2} r^{*}(d+k) r(d+k+N / 2) \\
R(d)=(1 / 2) \sum_{k=1}^{N-1}|r(d+k)|^{2}
\end{gathered}
$$

where $s_{k}$ is the PN sequence weighted factor for the $k^{t h}$ sample of the original preamble, $N$ is the FFT length and $M(d)$ is given by (5)

\section{E. Kang et al.}

Kang et al. designed a preamble pattern independent technique, which uses the principle behind the preamble structures employed to make the impulsive timing metric characteristic. In this technique, the correlation sequence of the preamble $C$ is derived as $C=B^{*} \circ B^{n}$, where "०" represents the Hadamard product, $B$ denotes the given preamble of length $N$ and $B^{n}$ denotes the circular shift of vector $B$ by an amount equal to $n$. Autocorrelation of $C$ has an impulsive response at the optimum value of $n . P(d), R(d)$ and $M(d)$ are given as [11]

$$
\begin{gathered}
P(d)=\Re\left\{r^{*}(d) \circ r^{n}(d)\right\}_{p}+\Im\left\{r^{*}(d) \circ r^{n}(d)\right\}_{q} \\
R(d)=\left\|\Re\left\{r^{*}(d) \circ r^{n}(d)\right\}\right\|+\left\|\Im\left\{r^{*}(d) \circ r^{n}(d)\right\}\right\| \\
M(d)=|P(d)|^{2} /(R(d))^{2}
\end{gathered}
$$

where $r(d)$ is the received vector of length $N$. The vectors $p$ and $q$ indicate the sign vector of $C$.

F. Yi et al.

This technique uses conjugate symmetric halves in the training symbol by interposing zeros as the guard band in the frequency domain form of the preamble. $P(d)$ and $R(d)$ are given as [8]

$$
\begin{gathered}
P(d)=\sum_{k=1}^{N / 2-1} r(m+k) r(m+N-k) \\
R(d)=\sum_{k=1}^{N / 2-1}|r(m+k)|^{2}
\end{gathered}
$$

where $N$ is the FFT length and $M(d)$ is given by (5).

\section{Proposed Timing Synchronization Technique}

We propose to use CAZAC sequence to design the training symbol which is significant for having unit PAPR [15]. Since OFDM signal has a large dynamic range with very high PAPR, non-linear power amplifiers at front-end clip OFDM signal, degrading its BER performance. Hence, power amplifiers are forced to work in their linear region but with CAZAC sequence having constant amplitude for training symbol, less back-off for power amplifiers is needed [16].

In our technique, we create eight identical portions of the training symbol by repeating the FFT of quarter length CAZAC sequence in all the portions. Eight identical segments help to provide steeper fall off of the Timing Metric from the strongest correlation point. To counteract the plateau of S\&C [4], we multiply each identical portion of Training Symbol by PN sign +1 or -1 for the described scheme. The correlation $P(d)$, the received energy $R(d)$ and the timing metric $M(d)$ are given as

$$
\begin{gathered}
P(d)=\sum_{k=0}^{L-2} b(k) \sum_{m=0}^{M-1} r^{*}(d+k M+m) r(d+(k+1) M+m) \\
R(d)=\sum_{i=0}^{M-1} \sum_{k=0}^{L-1}|r(d+i+k M)|^{2} \\
M(d)=\left(\frac{L}{L-1} \frac{|P(d)|}{R(d)}\right)^{2}
\end{gathered}
$$


where $b(k)=p(k) p(k+1)$ for $k=0,1, \ldots, L-2, p(k)$, $k=0,1, \ldots, L-1$, denote the signs of the repeated parts of the training symbol, $L$ is the number of identical portions, i.e., 8 and $M$ is the number of samples in each portion, i.e., 4 .

\section{Results}

In this section, we compare the performance of the proposed algorithm and the six existing algorithms in terms of timing metric and BER performance. The simulations are carried out in MATLAB. The key parameters for of OFDM system are given in Table I. Results are simulated for ISI Rayleigh faded channel with interference accumulating till $40 \%$ of cyclic prefix portion of adjacent symbol. A 7 tap multipath channel is considered and since a Rayleigh faded channel has an exponential power delay profile so the ratio of first Rayleigh fading tap to the last Rayleigh fading tap is set to $24 \mathrm{~dB}$.

TABLE I

OFDM SIMULATION PARAMETERS

\begin{tabular}{|c|c|}
\hline Number of sub-carriers & 32 \\
\hline Guard Interval $(\mathrm{CP})$ & $25 \%$ of preamble \\
\hline Modulation Scheme & BPSK \\
\hline Channel Encoding & $1 / 2$ Rate Convolutional Encoding \\
\hline$\lambda_{c}$ [see $\left.(3)\right]$ & 3 \\
\hline$\lambda_{f}$ [see $\left.(3)\right]$ & 1 \\
\hline
\end{tabular}

\section{A. Timing Metric}

We evaluate the Timing Metric for all the algorithms by using 1024 point training symbol (to acquire clear and smooth trends for the Timing Metric) with 128 samples cyclic prefix. Random 384 binary phase shift keying (BPSK) modulated samples are appended at the start to get the conformity of algorithm at the actual timing point. The actual starting point results to be $513^{\text {th }}$ sample $(384+128+1)$ as shown in Fig 2 . Timing Metric for $\mathrm{S} \& \mathrm{C}$ results in a plateau in ISI free part due to cyclic prefix. Ren et al., Yi et al., Park and Cheon and Kang et al. have impulsive timing metric. A roll-off type of correlation is the result for Minn et al.'s and proposed techniques as shown in Fig 2.

\section{B. MSE and BER results with Coarse Timing}

Fig. 3(a) shows the MSE of the timing offset verses Signal to Noise Ratio (SNR) (dB) for the proposed and six techniques with coarse timing only. The result for $S \& C$ technique is the worst and is not shown since it has the greatest uncertainty in the calculated timing offset point due to plateau in the timing metric. Minn et al.'s technique performs the best while the proposed technique is within $44 \%$ of Minn et al.'s MSE at moderate to high SNR. Fig. 3(b) shows the corresponding result for BER verses SNR. For the BER results, we see that $\mathrm{S} \& \mathrm{C}$ technique outperforms other algorithms because the mean of the plateau is taken as starting point of OFDM symbol, which acts as pre-advancement for dispersive channels. Actually there is no unique timing point that can be fixed. As long as the data stream is decoded starting from ISI free part, it can be recovered. The performance of the proposed algorithm is degraded as it is not designed to work in the absence of fine timing because of the steep roll-off of the timing metric.

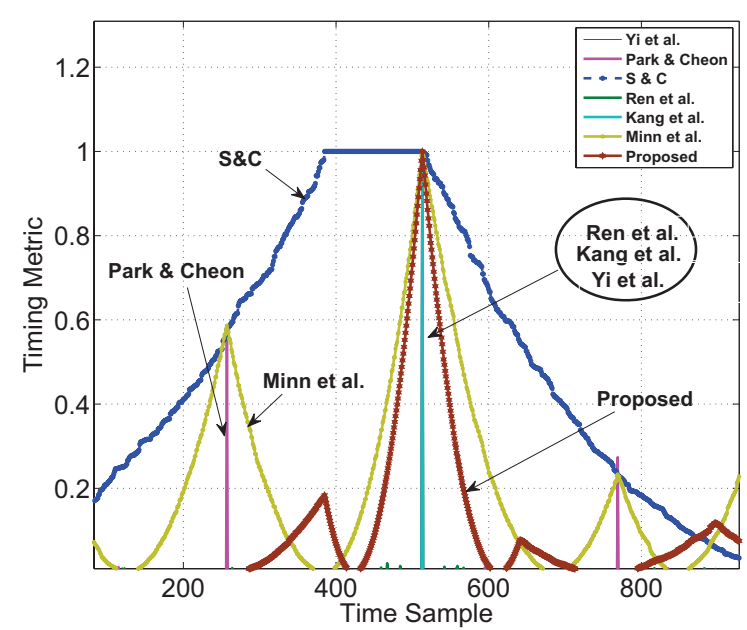

Fig. 2. Timing Metric plot for all the synchronization algorithms with $513^{t h}$ sample as the correct starting point. (Calculated from $M(d)$ of all the algorithms).

\section{MSE and BER results with Fine Timing}

Fig. 4 shows the (a) MSE of the timing offset and (b) BER verses SNR for the proposed and six techniques with both coarse and fine timing. We can see that the proposed technique outperforms Minn et al.'s MSE and the improvement is approximately $70 \%$ at high SNR. BER performance for $\mathrm{S} \& \mathrm{C}$ deteriorates in fine timing because timing point is already advanced when the mean of plateau is taken. Hence further pre-advancement in fine timing make the estimation out of the ISI free part resulting in absolute error. Comparing Fig. 3(b) and Fig. 4(b), we can see that BER performance with fine timing is better for every algorithm than with only coarse timing. S\&C method is efficient with only coarse timing block so it provides the best with least complexity. With fine timing, BER performance of proposed technique is very close to Minn et al.'s technique.

\section{CONCLUSION}

In this paper, we have provided a comprehensive analysis of different proposed timing offset algorithms and their effect on performance of OFDM system under severe frequency selective Rayleigh fading. We have computed the results for BER and MSE performance of all the algorithms with combined timing synchronization and channel estimation and thoroughly analyzed them. We have analyzed the performance with both coarse and fine timing and shown that BER performance with fine timing is better for every algorithm than with only coarse timing. We have shown that Minn et al.'s technique has better performance in both cases and S\&C method is efficient with only coarse timing, providing best result with least complexity. We can conclude that as long as the data stream is decoded starting from ISI free part, it can be recovered. Also, MSE performance should not be the only metric to be investigated. In fact BER performance with whole receiver design correctly demonstrates the worth of any algorithm. Finally, we have proposed a synchronization technique which uses fine timing and is robust to highly dispersive channels with BER performance comparable to the best case. 


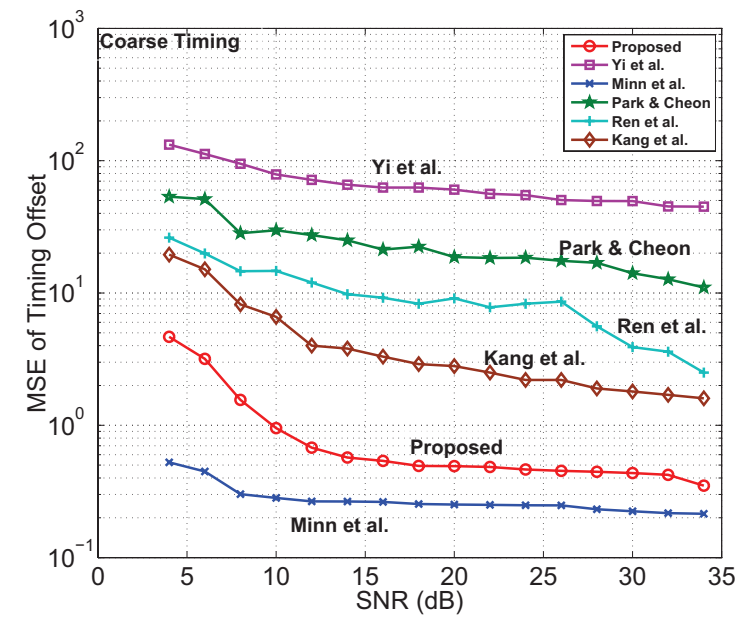

(a)

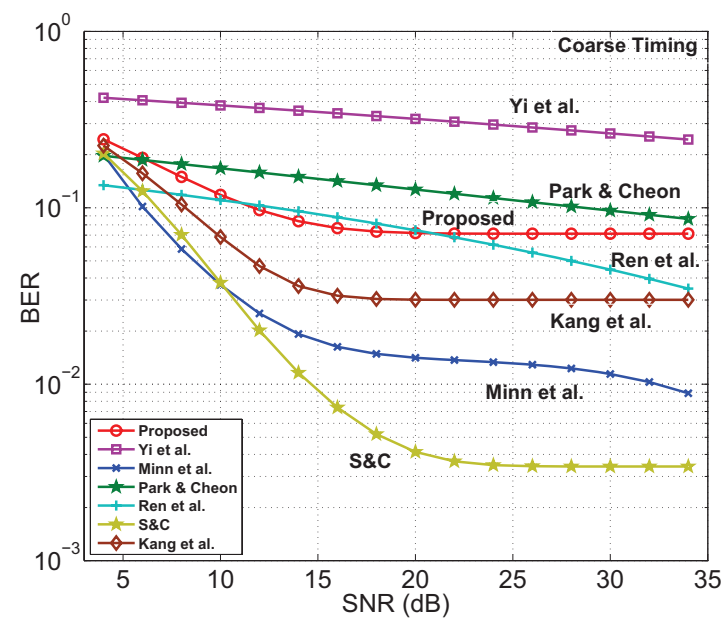

(b)

Fig. 3. (a) MSE of Timing offset estimation using coarse timing with SNR. (b) BER vs SNR plot using coarse timing.

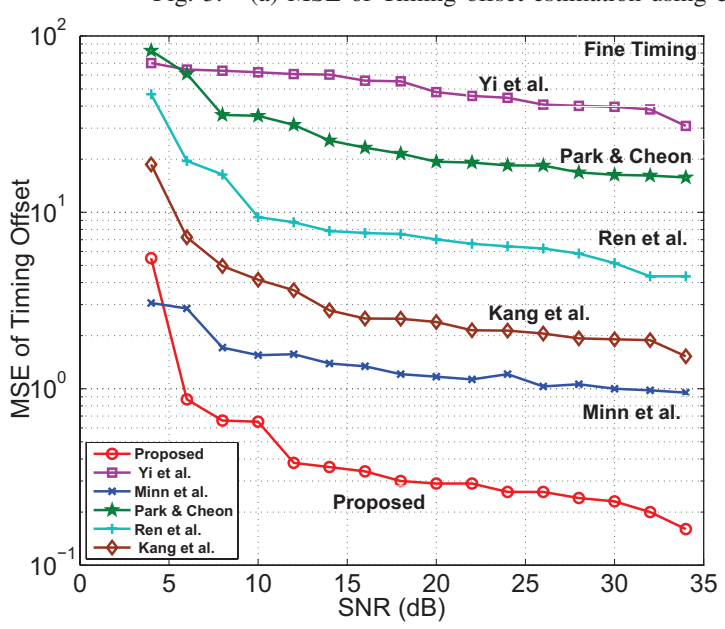

(a)

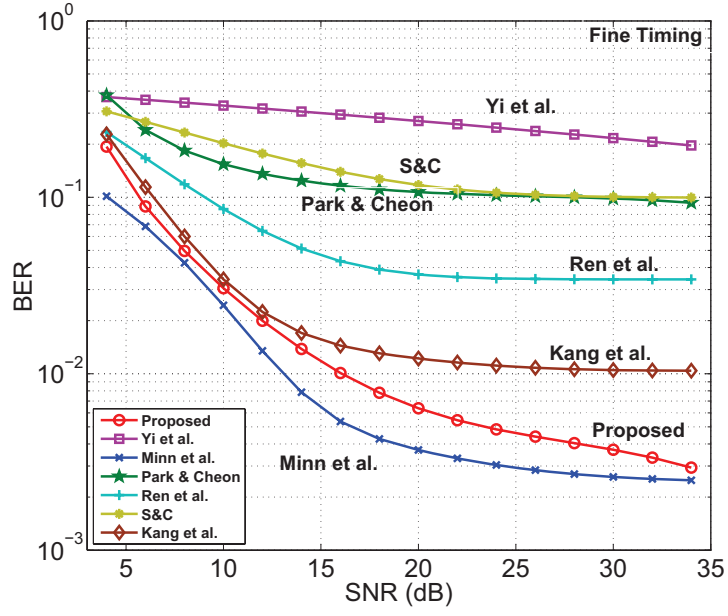

(b)

Fig. 4. (a) MSE of Timing offset estimation using fine timing with SNR. (b) BER vs SNR plot using fine timing

\section{REFERENCES}

[1] R. Prasad, OFDM for wireless communication systems. Artech House, 2004.

[2] M. Speth, S. A. Fechtel, G. Fock, and H. Meyr, "Optimum Receiver Design for Wireless Broad-Band Systems using OFDM Part-I," IEEE Trans. Commun., vol. 47, pp. 1668-1677, Nov. 1999.

[3] Y. Mostofi and D. C. Cox, "A Robust Timing Synchronization Design in OFDM Systems - Part 1: Low Mobility Case," IEEE Trans. Wireless Commun., vol. 6, pp. 4329-4339, Dec. 2007.

[4] T. M. Schmidl and D. C. Cox, "Robust Frequency and Timing Synchronization for OFDM," IEEE Trans. Commun., vol. 45, pp. 1613-1621, Dec. 1997.

[5] H. Minn, V. K. Bhargava, and K. B. Lataief, "A Robust Timing and Frequency Synchronization and Channel Estimation for OFDM," IEEE Trans. Wireless Commun., vol. 2, pp. 822-839, Jul. 2003.

[6] B. Park and H. Cheon, "A Novel Timing Estimation Method for OFDM systems," IEEE Commun. Lett., vol. 7, pp. 239 - 241, May 2003.

[7] G. Ren, Y. Chang, H. Zhang, and H. Zhang, "Synchronization Method Based on a New Constant Envelop Preamble for OFDM Systems," IEEE Trans. Broadcast., vol. 51, pp. 139-143, Mar. 2005.

[8] G. Yi, L. Gang, and G. Jianhua, "A Novel Timing and Frequency Synchronization Scheme for OFDM Systems," in Proc. WiCom, Sep. 2007, pp. 393 - 397.
[9] J. V. de Beek, M. Sandell, and M. Isaksson, "Low-complex frame synchronization in OFDM systems," in 4th IEEE International Conf. Universal Pers. Commun., 1995, pp. 982-986.

[10] M. Hsieh and C. Wei, "A low-complexity frame synchronization and frequency offset compensation scheme for OFDM systems over fading channels," IEEE Trans. Veh. Technol., vol. 48, pp. 1596-1609, Sep. 1999.

[11] Y. Kang, D. Ahn, and S. Kim, "Preamble Pattern Independent Timing Estimation for OFDM Systems," IEEE Electronics Lett., vol. 44, pp. 365 - 366, Feb. 2008.

[12] H. Bolcskei, "Blind estimation of symbol timing and carrier frequency offset in wireless OFDM systems," IEEE Trans. Commun., vol. 49, pp. 288-299, Jun. 2001.

[13] B. Park, H. Cheon, E. Ko, C. Kang, and D. Hong, "A blind OFDM synchronization algorithm based on cyclic correlation," IEEE Signal Process. Lett., vol. 11, pp. 83-85, Feb. 2004.

[14] H. Minn, V. K. Bhargava, and K. B. Letaief, "A Combined Timing and Frequency Synchronization and Channel Estimation for OFDM," IEEE Trans. Commun., vol. 54, pp. 416-422, Mar. 2006.

[15] Y. Wen, W. Huang, and Z. Zhang, "CAZAC sequence and its application in LTE random access," in IEEE Information th. Workshop, 2006.

[16] S. B. Slimane, "Reducing the Peak-to-Average Power Ratio of OFDM Signals Through Precoding," IEEE Trans. Veh. Technol., vol. 56, no. 2, pp. 686-695, Mar. 2007. 